\title{
A case of type III hyperlipoproteinaemia studied by acrylamide gel gradient electrophoresis
}

\author{
M. Y. CHEW, J. M. GUTMAN ${ }^{1}$, AND J. GREEN \\ From the Biochemistry Department, Repatriation General Hospital, Heidelberg West, Victoria 3081 \\ and Austin Hospital, Heidelberg, Victoria 3084, Australia
}

SUMMARY A case of type III hyperlipoproteinaemia has been investigated before and after treatment, and the unusual serum lipoprotein patterns obtained by molecular exclusion or pore limit electrophoresis on acrylamide gradients have been compared.

In their review of current diagnostic techniques in type III hyperlipoproteinaemia, Albers et al.(1977) state that 'the combination agarose-polyacrylamide gel electrophoresis system was not effective'. This technique, however, uses the discrepancy (sic) between the appearance of low density lipoprotein (LDL) or $\beta$ lipoprotein on polyacrylamide and agarose gel electrophoresis as a means of diagnosis (Masket et al., 1973). Molecular exclusion electrophoresis on acrylamide gradients (Green, 1976) should display the very low density (VLDL)-LDL spectrum in order of molecular size.

\section{Material and methods}

\section{ELECTROPHORESIS}

Separation of serum lipoproteins prestained with Sudan Black B was performed on acrylamide gradients, as described by Green (1976), with the following modifications. $0.13 \mathrm{ml}$ of $3 \mathrm{M}$ TRIS- $\mathrm{HCl}$, pH 8.9, was added to $4 \mathrm{ml}$ of the propane-1, 2-diol Sudan Black solution to minimise early cathodal migration. Gel dimensions are adjusted to suit different batches of acrylamide and BIS (Merck); in this case, the dimensions, concentrations, and cross-linkages used were $-1.8 \mathrm{~cm}$ of $7 \mathrm{~g} \%(2.5 \%)$, $1.6 \mathrm{~cm}$ of $5 \mathrm{~g} \%(2.25 \%), 1.6 \mathrm{~cm}$ of $4 \mathrm{~g} \%(2.25 \%)$, $0.75 \mathrm{~cm}$ of $3.5 \mathrm{~g} \%(1 \%)$, and $1 \mathrm{~cm}$ of $1.4 \mathrm{~g} \%(1 \%)$. All gradients are now formed with a peristaltic pump (Quickfit), delivering 1.3-1.6 $\mathrm{ml} / \mathrm{min}$ through a 21 gauge needle held just below the rising meniscus. The porosity of the air interface should be equivalent

${ }^{1}$ Present address: St. Vincent's Hospital, Melbourne, Australia.

Received for publication 13 September 1977 to that of a $2 \mathrm{~g} \%$ gel, $2 \cdot 25 \%$ cross-linked. Catalysts should be adjusted to give a gelation time of about 13 minutes at $\mathrm{RT}$.

Cellulose acetate electrophoresis was performed according to Kohn (1961), using Gelman Sepraphore III membranes, and agarose gel electrophoresis on EEL-Corning prepared gels using the makers' instructions.

\section{ULTRACENTRIFUGATION}

This was performed according to Hatch and Lees (1968) in a Beckman L5-75 ultracentrifuge. Serum, $1 \mathrm{ml}$ was diluted to $5 \mathrm{ml}$ to give densities (d) of 1.006 , $1.020,1.040$, and $1.060 \mathrm{~g} / \mathrm{ml}$ according to Havel et al. (1955). The upper layer of each supernatant and lower layer of each infranatant were aspirated and stained.

\section{Clinical}

The patient was a woman of 26 years under observation since May 1977 for angina of effort. She was observed to have subcutaneous xanthomata and yellowing of her palmar creases. The serum cholesterol values ranged from 14 to $10 \mathrm{mmol} / \mathrm{l}$ and triglycerides from $7 \cdot 3$ to $5.6 \mathrm{mmol} / \mathrm{l}$. Her serum was examined in this laboratory on 21 June 1977, and treatment with Atromid S, $3 \mathrm{~g} /$ day, was begun on 22 June. She had a coronary bypass operation on 12 July. On 25 July her serum cholesterol was $7 \cdot 1 \mathrm{mmol} / \mathrm{l}$ and triglycerides $3 \cdot 2 \mathrm{mmol} / \mathrm{l}$.

\section{Results}

The patient's gel pattern before treatment is shown in Figure 1. For comparison, a gel pattern with no visible material between VLDL and LDL and 


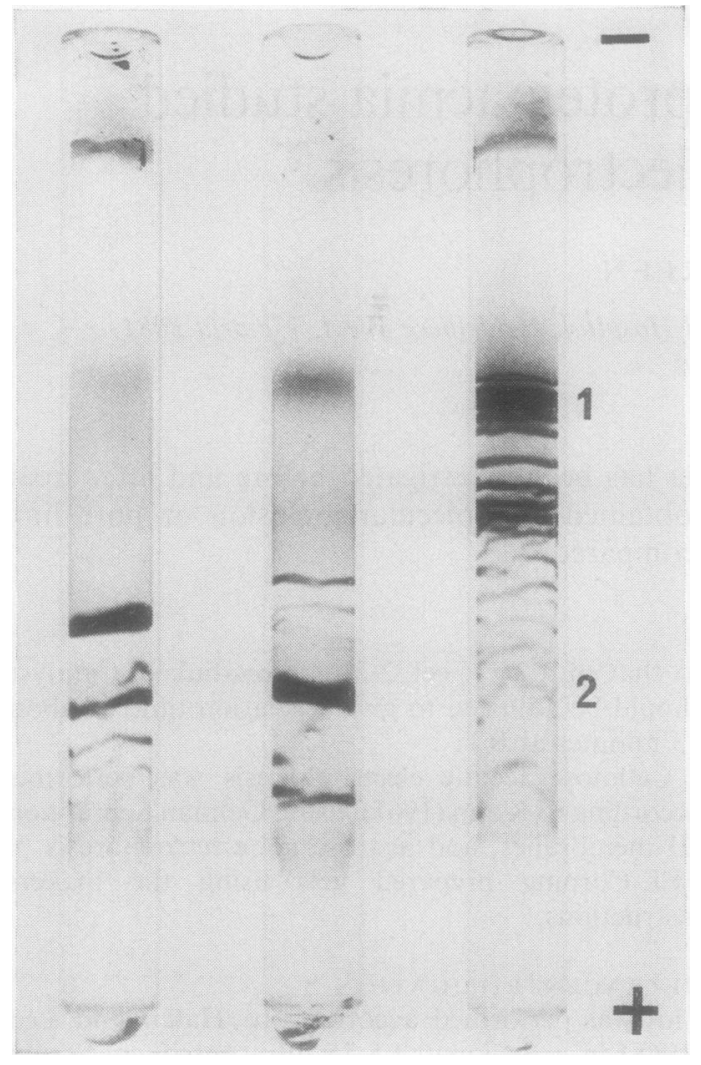

Fig. 1 The patient's gel pattern is shown on the right: 1 indicates the VLDL zone, and 2 the $L D L$ zone. The other two patterns are shown for comparison, as described in the text.

one with a 'central band' are included. The most obvious feature of the patient's pattern is the presence of several dense, well-defined bands just below the VLDL zone, which is itself increased and resolved. There is some unresolved material in the middle of the gel, and also in the LDL zone. All sections of the gel pattern gave a strong reaction with $\beta$ lipoprotein antiserum (Hoechst) on Laurell rocket electrophoresis. On cellulose acetate electrophoresis the appearance conformed to the description of the 'broad $\beta$ ' pattern. There was no stained material in the $\beta$ position, but the abnormal band began about $2 \mathrm{~mm}$ on the anodal side. The pre- $\beta$ band was increased and distinct from the abnormal band, and $a$ lipoprotein diminished.

The gel pattern after treatment is shown in Fig. 2, together with the patterns from supernatants and infranatants of d 1.006, 1.020,1.040, and 1.060. Although resolution tends to be different after ultracentrifugation, the overall pattern is clear. On cellulose acetate and agarose gel electrophoresis, stained material was present in the normal $\beta$ position and the previous abnormal band could not be detected. Stained material like that in the $\beta$ position was found in the supernatant fromd 1.020 , but not in that from d 1.006; its anodal mobility had increased after ultracentrifugation. The ratio of cholesterol to triglyceride in the supernatant from d 1.006 was 0.25 , and in that from $d 1.020,0.5$. The infranatant from d 1.006 showed traces of stained material in the pre- $\beta$ position; this may be linked to the faint but defined band in the centre of the gels $\mathrm{I} 2$ and $\mathrm{I} 3$ in Figure 2.

\section{Discussion}

The diagnosis of type III hyperlipoproteinaemia presented no problems in this case, and the interest lies in the additional information gained from the acrylamide gel patterns. Theoretically, components are displayed in order of molecular size, and the abnormal bands close to VLDL in position represent components close to it in size. In our experience, defined bands are unusual in this region and have been seen in other hyperlipaemias only when other defined components were present between this position and the LDL zone, and VLDL was increased.

The unusual appearance of 'LDL' in the infra-

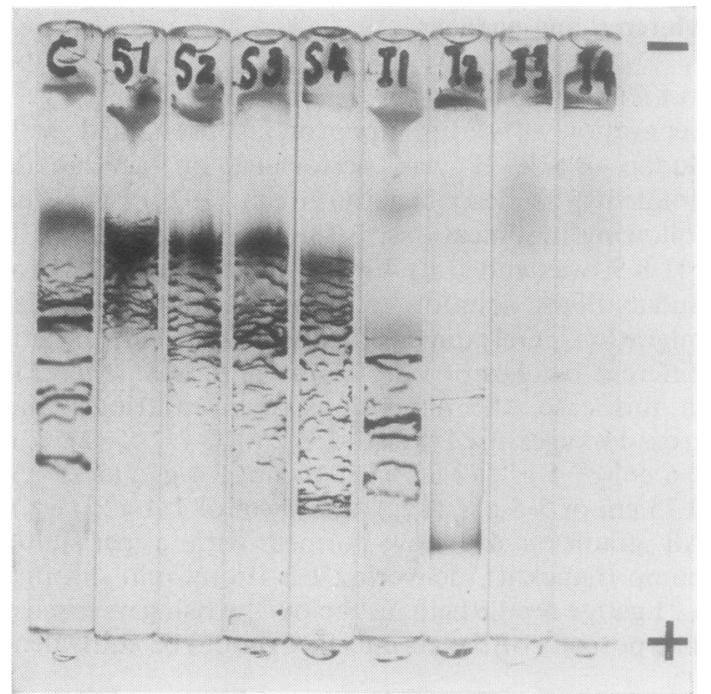

Fig. 2 Gel patterns after treatment: $C$-control whole serum. S1 to $S 4$ are supernatants of $d 1 \cdot 006,1 \cdot 020$, $1 \cdot 040$, and $1 \cdot 060 ; 11$ to $I 4$ are the corresponding infranatants. 
natant from d 1.006 and its unusual behaviour at d 1.020 - part removed from the infranatant, part appearing in the supernatant-contrasts with the presence of ' $\beta$ lipoprotein' on zone electrophoresis of whole serum and the infranatant from d 1.006. It suggests that this does not represent normal $\beta$ lipoprotein (Utermann et al., 1975).

We are glad to acknowledge financial support from the Department of Veterans' Affairs.

\section{References}

Albers, J. J., Warnick, G. R., and Hazzard, W. R. (1977). Type III hyperlipoproteinaemia: a comparative study of current diagnostic techniques. Clinica Chimica Acta, 75, 193-204.

Green, J. (1976). Lipoprotein pre-staining: comparison of nitroblue tetrazolium and Sudan Black B. Clinica Chimica Acta, 66, 295-301.
Hatch, F. T., and Lees, R. S. (1968). Practical methods for plasma lipoprotein analysis. Advances in Lipid Research, 6, 1-68.

Havel, R. J., Eder, H. A., and Bragdon, J. H. (1955). The distribution and chemical composition of ultracentrifugally separated lipoproteins in human serum. Journal of Clinical Investigation, 34, 1345-1353.

Kohn, J. (1961). A lipoprotein staining method for zone electrophoresis. Nature, 189, 312-313.

Masket, B. H., Levy, R. I., and Fredrickson, D. S. (1973). The use of polyacrylamide gel electrophoresis in differentiating Type III hyperlipoproteinaemia. Journal of Laboratory and Clinical Medicine, 81, 794-802.

Utermann, G., Jaeschke, M., and Menzel, J. (1975). Familial hyperlipoproteinaemia type III: deficiency of a specific apolipoprotein (apo E-III) in the very-lowdensity lipoproteins. FEBS Letters, 56, 352-355.

Requests to Dr John Green, Biochemistry Department, Repatriation General Hospital, Heidelberg West, Victoria 3081, Australia. 